\title{
LA SUSPENSIÓN DE PROCESOS PENALES COMO MEDIDA CAUTELAR EN JUICIOS ARBITRALES DE INVERSIÓN INTERNACIONAL ANTE EL CIADI: ¿VULNERACIÓN DE SOBERANÍA?
}

\author{
CRIMINAL PROCEDURE SUSPENSION AS PREVENTIVE \\ MEASURE IN INTERNATIONAL INVESTMENT \\ ARBITRATION: A HARM TO STATE SOVEREIGNTY?
}

\begin{tabular}{|c|c|}
\hline \multicolumn{2}{|c|}{ ARTÍCULO INÉDITO DE INVESTIGACIÓN } \\
\hline CÓMO CITAR ESTE ARTÍCULO (CHICAGO) & $\begin{array}{l}\text { Hudson Herranz, Lukas, “La suspensión } \\
\text { de procesos penales como medida caute- } \\
\text { lar en juicios arbitrales de inversión inter- } \\
\text { nacional ante el CIADI: ¿Vulneración de } \\
\text { soberanía?". Revista de Derecho Aplicado } \\
\text { LLM UC } 4 \text { (20I9). } \\
\text { doi: } \text { I0.77 }_{4} / \text { rda.o.\{.26r6 }\end{array}$ \\
\hline REVISTA DE DERECHO APLICADO LLM UC & $\begin{array}{l}\text { Número } 4 \\
\text { Diciembre } 20 \text { I9 } \\
\text { ISSN: } 245^{2-4344}\end{array}$ \\
\hline & $\begin{array}{l}\text { Recepción: } 3 \text { de septiembre, } 2019 \\
\text { Aceptación: I7 de noviembre, } 2019\end{array}$ \\
\hline
\end{tabular}




\section{Resumen}

El presente trabajo analiza si las medidas cautelares de suspensión de un proceso penal ordenadas por un tribunal arbitral de inversión internacional sujeto al Convenio CIADI vulneran la soberanía de un país. En nuestra opinión, si bien es una medida excepcional, creemos que la respuesta es negativa, toda vez que, conforme la jurisprudencia analizada, de manera tácita los tribunales arbitrales son proclives a su suspensión en casos en que el Estado utiliza dichos procesos para afectar los derechos del demandante en el proceso, o abusa del mismo para fines diversos.

Palabras clave: Arbitraje inversión internacional, medida cautelar, suspensión del proceso, abuso procesal, soberanía.

\section{Abstract}

This paper seeks to analyze whether an international arbitration tribunal in international investment trial, ordering the provisional measure of suspending a domestic criminal process, harms the sovereignty of the defendant State. In our opinion, although they are exceptional measure, it does not. Pursuant the analyzed case-law, the tribunals are implicitly inclined to suspend these proceedings when they affect the claimant's rights, or when the State abuses of those proceedings seeking other interests than those truth to the criminal process.

Keywords: International investment arbitration, preventive measure, suspended procedure, procedural abuse, sovereignty. 


\section{Lukas Hudson Herranz}

Lukas Hudson Herranz

Prado \& Aylwin Abogados

Santiago, Chile

lhudson@pradoaylwin.cl

Lukas Hudson Herranz

Prado \& Aylwin Abogados

Santiago, Chile

Ihudson@pradoaylwin.cl
Lukas Hudson Herranz es abogado de la Universidad de Chile, LLM in Comparative European Private Law, The University of Edinburgh, y Diplomado en Derecho Público Económico de la Universidad de Chile. Actualmente es profesor ayudante de Derecho Procesal en la Universidad de Chile. Ha publicado el ensayo "Rol de la Corte Suprema y naturaleza del recurso de reclamación en libre competencia: Hacia qué modelo de tribunal aspiramos" (en Reneé Rivero Hurtado y Juan Carlos Marín González, Reformas Necesarias a la Justicia. Tomo 2. Santiago: Librotecnia, 2018). Actualmente es asociado senior del área de litigios y arbitrajes en Prado \& Aylwin Abogados.

Lukas Hudson Herranz is a lawyer from the University of Chile, LLM in Comparative European Private Law, The University of Edinburgh, and a Diploma in Public Economic Law from the University of Chile. $\mathrm{He}$ is currently an Assistant Professor of Procedural Law at the University of Chile. He has published the essay "Rol de la Corte Suprema y naturaleza del recurso de reclamación en libre competencia: Hacia qué modelo de tribunal aspiramos" (in Reneé Rivero Hurtado and Juan Carlos Marín González, Reformas Necesarias a la Justicia. Volume 2. Santiago: Librotecnia, 2018). He is currently a senior associate in the litigation and arbitration area at Prado \& Aylwin Abogados. 


\section{INTRODUCCIÓN}

Los procedimientos arbitrales de inversión internacional son medios de solución de controversias entre inversionistas privados de naciones extranjeras respecto de países anfitriones. Amparados en tratados de inversión internacional bilaterales, denominados bilateral investment treaties (BIT), tratados multilaterales, denominados multilateral investment treaties (MIT), o en otros instrumentos internacionales de inversión o comercio como tratados de libre comercio, los Estados deben respetar los derechos de inversionistas extranjeros garantizando derechos mínimos, como el libre acceso, igual trato en los mercados y respeto al derecho de propiedad.

Dichos tratados son verdaderos "contratos" entre los inversionistas y los Estados, cuyo fin es garantizar que las inversiones se sometan a un mínimo de protección internacional $^{1}$. Luego, la vulneración de alguno de esos derechos por el Estado receptor de las inversiones habilita la gran mayoría de las veces a los inversionistas a llamar a arbitraje para la solución de la controversia, el cual es el principal medio de defensa de la parte afectada. Si bien existen varios cuerpos normativos de arbitraje, como el Reglamento de Arbitraje de la Cámara Internacional de Comercio (ICC), o el Reglamento de Arbitraje de la Comisión de Naciones Unidas para el Derecho Mercantil Internacional (UNCITRAL), muchas veces son sometidos al Convenio y Reglamento ante el Centro Internacional de Arreglo de Diferencias relativas a Inversiones, que es el cuerpo normativo preferido por inversionistas para solucionar los conflictos de inversión extranjera.

Cuestión de estudio son las medidas cautelares que dichos tribunales arbitrales pueden decretar. Las medidas cautelares son gestiones instrumentales cuyo fin es garantizar el resultado de un procedimiento ${ }^{2}$, las que tienen como principal propósito el asegurar el cumplimiento de una posible sentencia definitiva. En el caso de los arbitrajes internacionales sometidos a la normativa del Convenio CIADI, si bien la finalidad de las medidas cautelares es preservar los derechos de las partes ${ }^{3}$, ello es solo otra fórmula fundada en el principio rector como es el congelamiento de la situación jurídica de las partes durante el proceso, para prevenir posibles fraudes relativos al cumplimiento de la sentencia definitiva ${ }^{4}$.

1 Zachary Douglas, "The Hybrid Foundations of Investment Treaty Arbitration". The British Yearbook of International Law 74, n. ${ }^{\circ}$ I (noviembre 2004): I52-I55.

2 Gonzalo Cortez Matcovich, "Contribución al estudio de las medidas cautelares previas a la demanda en el proceso civil chileno" Revista del Derecho 30, n. $^{\circ}$ I (20I7): 236.

3 Chin Leng Lim, Jean Ho y Martins Paparinskis, International Investment Law and Arbitration. (Cambridge: Cambridge University Press, 2018), I82.

4 Lim, Ho y Paparinskis, International Investment, 182. 
Entre estas medidas, llama especial atención aquellas cuyo fin es la paralización de procesos penales que pudiesen incidir en su resultado. Como sabemos, el proceso penal es un ejemplo del poder soberano de un Estado, entendido como aquel poder supremo hospedado en él para organizarse como tal a su entera voluntad ${ }^{5}$. Si bien existen varias definiciones de soberanía, lo cierto es que van en la línea de evitar limitaciones a su ejercicio, salvo aquellos límites impuestos a sí mismos ${ }^{6}$.

Dicho lo anterior, zes posible decretar la suspensión de un proceso penal mediante una medida cautelar? ¿Inciden estos procesos en el resultado de un arbitraje de inversión internacional? ¿Cuál sería el fundamento o razón que lo permita? Luego, ¿es posible hablar de una intromisión en la soberanía de un país al ordenar mediante un tribunal internacional la suspensión del aparato jurisdiccional penal?

El punto en cuestión es saber qué tan legitimado se encuentra un tribunal arbitral para ordenar a un Estado, un sujeto del derecho internacional público por excelencia, a inhibirse en una actividad pública como la jurisdicción. Asimismo, cuál es el límite en el otorgamiento de una medida de esa complejidad. Nótese que decretar una medida de esta magnitud en cierto sentido limita el desenvolvimiento de un Estado y soslaya su normativa interna, lo que también permitiría la suspensión de dichos procesos en caso de ser necesario. El presente trabajo busca dar una respuesta sobre por qué y hasta dónde puede llegar un tribunal arbitral ante este tipo de solicitudes. En esencia, se busca un elemento unificador que pueda entregar luces de cuándo es viable este tipo de medidas cautelares y cuándo no es aconsejable decretarlas.

Por otra parte, este ensayo analiza las razones de la suspensión de algunos juicios penales por órdenes de tribunales arbitrales internacionales de inversión regidos bajo las leyes del Convenio CIADI, y si ello vulneraría el principio de soberanía nacional. Si bien la gran mayoría de estos fallos obedece a evitar mayores perjuicios en los derechos de las partes, a nuestro parecer existe un trasfondo común en la jurisprudencia del Convenio CIADI consistente en el abuso de las herramientas jurídicas otorgadas por el proceso penal doméstico para fines distintos a los dispuestos en esas leyes, lo que muchas veces haría necesaria dicha suspensión. La razón de revisar este fenómeno tiene directa relación con la expansión de las medidas cautelares como mecanismo de resguardo de los derechos de las partes en un litigio y el objeto del mismo, especialmente derechos garantizados en tratados internacionales que promueven la inversión y el resguardo a la propiedad privada. La pregunta que queremos responder es cuál es el límite de dicha pretensión. ¿Es la soberanía u otro elemento?

5 Lautaro Ríos Álvarez, "La soberanía, el poder constituyente, y una nueva constitución para Chile". Estudios Constitucionales I5 $_{5}$,.$^{\circ}{ }_{2}$ (20I7): I76-I77.

6 Georg Jellinek, Teoría general del Estado (Buenos Aires: Albatros, I978), $35^{6 .}$ 


\section{MEDIDAS CAUTELARES EN EL ARBITRAJE INTERNACIONAL DE INVERSIONES EXTRANJERAS}

Las facultades cautelares de los tribunales arbitrales de inversión extranjera se encuentran consagradas en la mayoría de los principales reglamentos arbitrales. Un ejemplo es el artículo 28 en del Convenio del ICC, en el que encontramos la facultad del tribunal arbitral de decretar y otorgar las medidas cautelares pertinentes, las que se decretarán "siempre que sean adecuadas y fundadas para el fin que son otorgadas". En el análisis que nos atañe, en los procedimientos arbitrales sujetos al CIADI, los artículos 47 del Convenio y 39 de su Reglamento establecen las facultades cautelares del tribunal, cuyo fin es preservar los derechos de las partes ${ }^{8}$.

Una pregunta previa sería por qué es legítimo este tipo de medidas. Nótese lo ya controversial que es ejecutar medidas intrusivas contra un Estado demandado como, es la incautación de bienes dentro de sus fronteras, infringiendo - o al menos restringiendoel principio internacional de soberanía e inembargabilidad de bienes de los Estados ${ }^{9}$. Es imposible soslayar que los embargos e incautaciones sobre bienes utilizados por el Estado muchas veces interfieren con los fines entregados por este último, como son satisfacer necesidades públicas o el buen funcionamiento del mismo. Por lo tanto, una deferencia de limitar estas medidas no sería extraña.

7 Artículo 28, Reglas de Arbitraje ante la Cámara Internacional de Comercio, párrafo I: “Salvo acuerdo de las partes en contrario, el tribunal arbitral podrá, desde el momento en que se le haya entregado el expediente, ordenar, a solicitud de parte, cualesquiera medidas cautelares o provisionales que considere apropiadas. El tribunal arbitral podrá subordinar dichas medidas al otorgamiento de una garantía adecuada por la parte que las solicite. Las medidas mencionadas deberán ser adoptadas mediante orden motivada o laudo, según el tribunal arbitral lo estime conveniente”.

8 Artículo ${ }_{47}$, Convenio de Centro Internacional de Arreglo de Diferencias relativas a Inversiones: "Salvo acuerdo en contrario de las partes, el Tribunal, si considera que las circunstancias así lo requieren, podrá recomendar la adopción de aquellas medidas provisionales que considere necesarias para salvaguardar los respectivos derechos de las partes”.

9 Para mayor información sobre inmunidad de los Estados, véase Malcolm Shaw, International Law (Cambridge: Cambridge University Press, 20I7), 522. En el mismo sentido: James Crawford, Brownlie's Principles of Public International Law (Oxford: Oxford University Press, 2012), 487. Sobre inmunidad de embargos sobre bienes estatales, véase Andrea Bjorklund, "State Immunity and the Enforcement of Investor-State Arbitral Awards". En International Investment Law for the 2Ist Century: Essays in Honour of Christoph Schreuer, de Christina Binder y otros (Oxford: Oxford University Press, 2009). 
Sin embargo, se ha aceptado que las medidas cautelares son una especial excepción procesal a dicha regla, cuyo fin es la abstención de las partes de ejecutar ciertas conductas que puedan causar un perjuicio tal que sea posteriormente irreparable ${ }^{10}$. No buscan una anticipación o presión para cumplir los intereses de una parte, sino simplemente la conservación de los derechos de las partes. Así lo ha entendido la jurisprudencia especializada del CIADI: en Biwater Gauff (Tanzania) Ltd con Tanzania ${ }^{11}$, se falló que las medidas cautelares buscan esencialmente evitar: i) cualquier perjuicio en términos de integridad del procedimiento; o ii) agravar la posición de alguna de las partes ${ }^{12}$. A pesar que este último juicio fue sometido a la normativa del CIADI, creemos que sus fundamentos podrían ser perfectamente análogos a la razonabilidad detrás de otros cuerpos arbitrales de inversión extranjera. Por ejemplo, en la normativa del Convenio del ICC, sin perjuicio de que tiene un mayor rango de acción, ya que pueden obtenerse "si son apropiadas", según las circunstancias $^{13}$. Sin perjuicio de lo anterior, y como toda medida cautelar, debe ser fundada de modo que evite al tribunal caer en arbitrariedades o desproporciones en su decisión.

Las medidas que pueden ser decretadas por el tribunal ante procedimientos sujetos a la Convención CIADI no se encuentran acotadas o restringidas a un listado, ya que es facultativo de las partes solicitar la medida que se ajuste a sus intereses, como también el tribunal arbitral goza de discreción para decretar cuál es el mecanismo idóneo para preservar los derechos alegados ${ }^{14}$. Así, las medidas pueden ir desde evitar la destrucción de documentos ${ }^{15}$, paralizar el pago de dineros ${ }^{16} \mathrm{y}$ hasta restringir la aplicación de normativa tributaria interna de un país ${ }^{17}$. A pesar de que existen varias medidas interesantes y

10 Tarcisio Gazzini y Robert Kolb, "Provisional Measures in ICSID Arbitration from 'Wonderland's Jurisprudence' to Informal Modification of Treaties", The Law \& Practice of International Courts and Tribunals $\mathrm{I} 6, \mathrm{n}^{\circ}{ }^{\mathrm{I}}$ (junio 20I7): I6I-I62.

11 Biwater Gauff (Tanzania) Ltd con Tanzania, caso CIADI ARB/०5/22, orden procesal 3, 29 de septiembre de 2006 .

12 Biwat er Gauff (Tanzania) Ltd con Tanzania, párrafo I35.

13 Dan Sarooshi, "Provisional Measures and Investment Treaty Arbitration". Arbitration International 29, n. $_{3}$ (septiembre $\left.20 \mathrm{I} 3\right): 370$.

14 María Fernanda Vásquez, "Arbitraje ante el CIADI: Aspectos relevantes y reflexiones sobre su operatividad" Ministerio de Justicia del Perú, https://www.minjus.gob.pe/wp-content/ uploads/20I7/04/ASPECTOS-RELEVANTES-CIADI-CHILE.pdf.

15 Abaclat y otros con República Argentina, caso CIADI ARB/o7/5, orden procesal II, 27 de junio de 20I2, párrafos I6-I7.

16 City Oriente con República de Ecuador, caso CIADI ARB/o6/2I, resolución a solicitud de demandante por medida cautelar, ig de noviembre de 2007 .

17 Sergei Paushok, CJSC Golden East Co. and CJSC Vostoknftegaz Co. con Gobierno de Mongolia, UNCITRAL, orden sobre medidas cautelares, 2 de septiembre de 2008. 
de difícil explicación, este estudio se acotará a las medidas llamadas a suspender juicios internos llevados ante el Estado demandado.

En efecto, existen diversas resoluciones dictadas conforme el Convenio CIADI en las que se decreta la suspensión de procedimientos, fundado genéricamente en la preservación de los derechos del demandante ${ }^{18}$. 2 Es posible aquello? Como sabemos, los tribunales son representantes de la soberanía estatal, en cuanto órganos encargados de solucionar conflictos entre las partes y evitar la autotutela. ¿Podría una medida de esta magnitud implicar alguna vulneración a la soberanía del Estado demandado? La pregunta es legítima toda vez que la suscripción de tratados de inversión internacional bilaterales o multilaterales no implica en modo alguno la renuncia de soberanía sobre bienes de un Estado, mucho menos en la entrega de servicios públicos o actividades propias de un Estado como defensa o la administración de justicia. Después de todo, el resultado de una medida como la aludida es la intromisión de un tribunal extranjero dentro de la esfera jurisdiccional de un Estado soberano. ¿Acaso ello es una restricción a su poder soberano?

¿Es la dictación de estas medidas una excepción razonable para el resguardo de los derechos del demandante inversionista o una medida que riñe con el principio internacional de inmunidad soberana?

Para dicho análisis, creemos necesario separar las medidas cautelares decretadas por estos tribunales según juicios civiles y juicios penales.

\section{I. Juicios civiles}

Una vez iniciado un arbitraje de inversión internacional, es común que las partes soliciten la suspensión de juicios civiles internos radicados en la jurisdicción del Estado demandado. Según lo dispuesto en el artículo 26 de la Convención CIADI, una vez constituido, se otorga jurisdicción exclusiva al tribunal arbitral para resolver los conflictos sometidos a su conocimiento ${ }^{19}$, así como la prohibición de cualquier recurso que atente contra su unidad jurisdiccional ${ }^{20}$. Por esta sencilla razón, los tribunales nacionales no pueden intervenir en un arbitraje de inversión extranjera ni tampoco ser utilizados para prevenir o evadir sus resultados.

Ahora bien, las razones anteriores pueden aglutinarse en torno al principio de litispendencia: no es posible mantener dos procedimientos en paralelo en los que se ejerce la misma acción o pretensión en cada juicio y entre las mismas partes, ya que existe

18 Jeffery Commission y Rahim Moloo, Procedural Issues in International Investment Arbitration (Oxford: Oxford University Press, 2018), 44 .

19 Commission y Moloo, Procedural Issues, 43.

20 Sarooshi, “Provisional Measures”, 37I. 
una triple entente entre ambos pleitos ${ }^{21}$. El problema nace en casos en que un tribunal nacional podría pronunciarse en forma anticipada sobre alguna de las pretensiones ventiladas en el arbitraje internacional ${ }^{22}$, en que el primero abarca total o parcialmente parte de la discusión ventilada en el tribunal arbitral, lo que podría producir sentencias contradictorias $^{23}$. En mi opinión, si las partes han sometido un conflicto a la jurisdicción de un tribunal arbitral internacional, no es posible iniciar un segundo procedimiento. Eso implica que, iniciado el procedimiento, salvo acuerdo expreso, las partes excluyen el litigio de la competencia de los tribunales locales, por lo que no pueden iniciarse nuevos procesos que influyan o contravengan el resultado del proceso arbitral ${ }^{24}$.

Así de hecho se falló en el caso Ceskoslovenska Obchdni Bank A.S. con República de Eslovaquia, cuando el tribunal arbitral accedió a suspender un procedimiento de quiebra doméstico fundado en que tenía directa injerencia en el fondo del asunto ${ }^{25}$. En otras palabras, un procedimiento en paralelo que infringía la integridad del arbitraje, o al menos hacía un pronunciamiento anticipado que afectara el procedimiento arbitral. Mismo razonamiento es hallado en Plama Consortium Limited con Republica de Bulgaria, cuando el demandante solicitó la suspensión de un procedimiento de quiebra de una empresa afiliada a la demandante. Si bien el tribunal denegó la paralización del procedimiento ante los tribunales búlgaros, ello se debió a que los derechos disputados en el arbitraje se relacionaban con la violación de un trato justo en las inversiones de la empresa demandante derivados del Tratado de la Carta de Energía, mientras que el procedimiento sometido a los tribunales búlgaros y objeto de la medida consistía en la liquidación de una empresa. Para el tribunal, eran juicios con derechos y peticiones completamente diversos ${ }^{26}$. Asimismo, las partes y pretensiones en ambos juicios eran distintos ${ }^{27}$.

En consecuencia, la suspensión de juicios civiles en arbitrajes de inversión extranjera es aceptada conforme sigue el principio de la litispendencia. Su otorgamiento no viene

21 Alejandro Romero Seguel, Curso de derecho procesal civil, tomo 3 (Santiago: Thomson Reuters, 20I5), I24.

22 Commission y Moloo, Procedural Issues, 43 .

23 Ignacio Ried Undurraga, "Tres cuestiones sobre la litispendencia en el proceso civil chileno". Revista de Derecho de la Pontificia Universidad Católica de Valparaíso 45 (segundo semestre 20I5): 2IO-2II.

24 Campbell McLachlan, Laurence Shore y Matthew Weiniger, International Investment Arbitration (Oxford: Oxford University Press, 20I7), 105.

25 Ceskoslovenska Obchdni Bank A.S. con República de Eslovaquia, caso CIADI ARB/97/4, decisión del tribunal sobre objeción de jurisdicción, 24 de mayo de i999, párrafo 9 .

26 Plama Consortium Limited con República de Bulgaria, caso CIADI ARB/०3/24, orden, 6 de septiembre de 2005 , párrafo ${ }_{4} \mathrm{I}$.

27 Plama Consortium Limited con República de Bulgaria, párrafo $4^{2}$. 
precisamente de las facultades cautelares del tribunal, sino de la prevención de mantener la continencia del procedimiento y evitar cualquier posible contradicción entre dos sentencias distintas. Así, el tribunal arbitral solamente accederá a su suspensión si los tribunales domésticos afectan el principio de integridad del tribunal o existe una anticipación del caso concreto. En palabras de Nieva, "hay que fijarse para decidir si dos procesos son iguales, es el punto central de la controversia, es decir, que se trate de la misma controversia sobre consecuencias jurídicas acerca de las mismas situaciones de hecho"28.

\subsection{Juicios penales}

Mayor complejidad ofrece la suspensión de procedimientos penales durante un procedimiento arbitral de inversiones extranjeras sometidos a la Convención CIADI. Debido a que el proceso penal persigue la imputación de actos susceptibles de responsabilidad penal, en principio no debiese intervenir o influir en los resultados de procesos arbitrales. Sin embargo, a pesar de ello, algunos tribunales arbitrales han decretado la suspensión de estos procesos criminales mediante medidas cautelares, fundados y regulados bajo la normativa del Convenio CIADI, para lo que exigen un umbral de prueba particularmente alto ${ }^{29}$.

Lo anterior muestra que este tipo de medidas sí se encuentra dentro de la competencia de los tribunales arbitrales de inversión internacional sujetos a esta normativa. Sin embargo, ¿cuál es el motivo de su otorgamiento? Para tratar de dar una posible respuesta, hemos seleccionado cinco casos en que los demandantes solicitan la suspensión de determinados procedimientos penales.

El primer caso es Tokios Tokelés con Ucrania, en el que se planteó por primera vez la posibilidad de paralizar un procedimiento penal. En este caso, la demandante, una empresa de imprenta y publicidad, solicitó la suspensión de un proceso penal seguido ante los tribunales ucranianos en contra de uno de los representantes principales de la empresa arguyendo que dicho proceso dañaría su prueba, entre ellos la declaración de su representante. Si bien la solicitud fue rechazada debido a que no se logró probar la necesidad de la medida - el proceso penal comenzó nueve meses antes de la solicitud de arbitraje, mas no fue solicitado en una anterior solicitud de medidas cautelares, lo que disminuyó su credibilidad y urgencia-, lo relevante del caso es que el tribunal declaró que tenía la suficiente competencia para paralizar procedimientos domésticos, "siempre y cuando la acción de la contraria se encuentre relacionada con la materia del procedimiento" ${ }^{30}$.

\footnotetext{
28 Jordi Nieva, Derecho procesal I: Introducción (Madrid: Marcial Pons, 20I4), 53.

29 Commission y Moloo, Procedural Issues, 44.
} 
Esto último implicó que el tribunal hipotéticamente pudo suspender un proceso penal llevado ante un Estado soberano si se cumplían los requisitos legales según el artículo 47 del CIADI.

Resultado distinto fue el obtenido en City Oriente Limited con República de Ecuador. El caso surgió a partir de la modificación unilateral de un contrato de producción de hidrocarburos entre City Oriente Limited y Ecuador, debido a la nueva Ley 2006-42 de Hidrocarburos, que aumentaba el pago de royalties a favor del Estado ecuatoriano. Resultado de este cambio, la empresa se opuso al pago de dicho aumento y, a su vez, el Estado ecuatoriano inició procedimientos criminales contra los ejecutivos de la empresa por el no pago de dichos impuestos ante el Ministerio Fiscal competente ${ }^{31}$. Ello hizo que City Oriente solicitara la suspensión de dichos procedimientos. Luego de ponderar los antecedentes, el tribunal arbitral finalmente ordenó su suspensión: por un lado, porque debía mantener el statu quo de los derechos de las partes por los posibles perjuicios que podría producir aquella situación ${ }^{32}$, pero principalmente por la intención del Estado ecuatoriano de presionar a la demandante por esa vía para pagar los impuestos reclamados ${ }^{33}$.

El tercer caso en análisis es el juicio Quiborax S.A., Non Metallic Minerals S.A. y Allan Fosk Kaplún con Bolivia. El conflicto comenzó por la decisión unilateral de Bolivia de revocar once concesiones mineras de propiedad de una sociedad cuyo principal accionista era Quiborax S.A., lo que llevó a una confiscación de sus inversiones por parte del Estado boliviano. En paralelo, Bolivia inició varios procedimientos penales contra personas relacionadas con Quiborax S.A. por una serie de delitos, desde supuesta falsificación de documentos societarios por parte de personeros relacionados a Quiborax S.A., hasta uso indebido de documentación falsa. La empresa solicitó la paralización de dichos procedimientos criminales, por cuanto el proceso penal inhibía su derecho de defensa y de prueba. El tribunal acogió la solicitud de la demandante y suspendió los procesos penales, fundado más que nada en que el proceso penal alteraba no solo el status quo de las partes y sus derechos - en esencia su derecho a la prueba, toda vez que el proceso mermaba la prueba de la demandante al inhibir la declaración de testigos o impedir la recolección de

Tokios Tokelés con Ucrania, caso CIADI ARB/०2/18, orden 3, resolución a solicitud de demandante por medida cautelar, 18 de enero de 2005. párrafo II. Misma opinión se puede encontrar en Commission y Moloo, Procedural Issues, 195.

Las denuncias fueron interpuestas tanto por un diputado de la República de Ecuador y por el abogado procurador general del Estado. Para mayor información: City Oriente con República de Ecuador, caso CIADI ARB/o6/2I, resolución a solicitud de demandante por medida cautelar, I9 de noviembre de 2007 , párrafos I5-18.

32 Commission y Moloo, Procedural Issues, ig6.

33 City Oriente con República de Ecuador, párrafos 63, 66. 
determinada documentación-, sino también por el supuesto móvil del Estado boliviano de iniciar el proceso penal al conocerse la intención del demandante de solicitar el procedimiento arbitral, todo ello con el fin de mejorar su situación procesal ${ }^{34}$.

El cuarto caso a destacar es el fallo en Nova Group Investments, B.V. con Romania, del año 2017. El caso consiste en una demanda interpuesta por una empresa de capitales holandeses, Nova, que demanda al Estado de Rumania por una serie de infracciones cometidas por la autoridad regulatoria financiera del país, la que habría forzado el proceso de liquidación de la sociedad y vehículo financiero del demandante, Astra Asigurări. En paralelo a dicho procedimiento, y antes del inicio del arbitraje, Rumania seguía un proceso de extradición en contra de uno de los representantes de la empresa, Alexander Amadescu, quien residía en el Reino Unido, a quien acusaba de sobornar a ciertos jueces que conocieron de los procesos de liquidación de Astra Asigurări. Su extradición surgió debido a la negativa de asistir a una citación para declarar en calidad de investigado, ante la sospecha de quedar detenido en Rumania.

Debido a este procedimiento de extradición, Nova solicitó una medida cautelar de congelamiento del proceso de extradición de Amadescu bajo el argumento de que era un testigo importante para el proceso arbitral. Asimismo, alegó que era la persona que dirigía la empresa, aportaba consejo para el caso y documentación como medios de prueba, por lo que extraditarlo atentaría contra su derecho a defensa y reproducir pruebas ${ }^{35}$. El tribunal arbitral decidió acoger la medida cautelar y ordenó al Estado rumano suspender el proceso de extradición. Las razones del tribunal arbitral se fundaron principalmente en el exclusivo conocimiento de Amadescu sobre sus compañías en Rumania, como también el hecho de que su testimonio era vital para saber el contenido de la información de las empresas de Nova en Rumania. Al ser además el único perseguido por el Estado rumano por los supuestos problemas financieros de Astra Asigurări, el tribunal concedió el hecho de que su persecución podría perjudicar los intereses de la demandante ${ }^{36}$.

Por último, cabe destacar el caso de Lao Holding con Laos. El juicio surgió a partir de una demanda interpuesta por una empresa de origen holandés contra la República Democrática Popular de Laos por una serie de conductas del Estado demandado, al ejecutar una expropiación de facto sobre sus inversiones. En concreto, el Estado anunció la aplicación

34 Quiborax S.A., Non Metallic Minerals S.A. y Allan Fosk Kaplún con Estado Plurinacional de Bolivia, caso CIADI ARB/o6/2, decisión sobre medidas provisionales, 26 de febrero de 2010 , párrafos II9-I2, ${ }_{4}{ }_{4}^{2-I} 43$.

35 Nova Group Investments, B.V. con Romania, caso CIADI ARB/r6/rg, decisión sobre medidas provisionales 7, 29 de marzo de 2017, párrafo I37.

36 Nova Group Investments, B.V. con Romania, párrafos 286-289. 
de una serie de impuestos sobre el rubro de sus inversiones - turismo y casinos de juego-, lo que expropiaría sus inversiones. Debido a la posibilidad cierta de que se apliquen aquellos impuestos y se pierdan las inversiones efectuadas, el demandante pidió la paralización de aplicar dichos impuestos y cualquier procedimiento que busque su cobro. E1 tribunal accedió a la petición ${ }^{37}$.

El punto de análisis es una solicitud del Estado demandado que busca modificar la medida cautelar solicitando al tribunal la autorización de promover ciertos procedimientos criminales contra personeros relacionados con la empresa demandante, toda vez que existen fundamentos que acusarían posibles sobornos de estas personas a funcionarios del Estado de Laos. Respecto de esta petición, si bien el tribunal arbitral ratifica el principio de que un Estado es libre de hacer las investigaciones y procedimientos correspondientes, advierte que deben ser iniciados de buena fe y respetando los derechos y pretensiones del demandante en el arbitraje ${ }^{38}$. En el caso en comento, la solicitud fue precisamente rechazada toda vez que el tribunal razonó que los procedimientos buscarían generar pruebas a favor de su teoría del caso, lo que generaría una clara ventaja indebida en contra del demandante ${ }^{39}$, junto con denunciar que los procesos criminales perjudicarían e inhibirían las declaraciones de los testigos de la parte demandante y podrían limitar el acceso a documentación relevante para su teoría del caso $^{40}$. Por estas razones, la solicitud fue rechazada.

Analizando los fallos en detalle, es posible identificar un principio general esgrimido por los tribunales arbitrales para la paralización de dichos procedimientos penales, consistente en la prohibición del uso del proceso penal para perjudicar la posición legal del inversionista demandante en el arbitraje. El artículo 47 del Convenio CIADI establece que las medidas cautelares deben ser decretadas buscando preservar los derechos de las partes, mientras que los procesos penales aparentemente fueron instrumentalizados con fines diversos, incluso llegando a dañar al demandante. Así, en City Oriente con Ecuador, la sentencia explica que los procesos penales buscaban presionar a la empresa para adoptar las medidas de la nueva ley de hidrocarburos ${ }^{41}$; mientras en Quiborax con Bolivia su uso indebido perjudicaba el derecho de prueba de la empresa al propagar temor en testigos o impedir el ejercicio de dicho derecho ${ }^{42}$. En el caso de Nova con Romania, si bien el proceso

37 Lao Holdings N.V. con República Democrática Popular de Laos, caso CIADI ARB (AF)/r2/6, decisión sobre medida cautelar, I7 de septiembre de 2013 .

38 Lao Holdings N.V. con República Democrática Popular de Laos, caso CIADI ARB (AF)/r2/6, decisión para modificar medida cautelar, 30 de mayo de 20I4, párrafo 25.

39 Lao Holdings N.V. con República Democrática Popular de Laos, párrafos 27-32.

40 Lao Holdings N.V. con República Democrática Popular de Laos, párrafos 40-42.

41 City Oriente con Ecuador, párrafos 62-63, 65-66. 
de extradición ya se encontraba iniciado, era evidente que su foco era mermar la prueba del demandante con miras a obtener ventajas indebidas de dicho proceso. En los tres casos, los procesos habrían sido utilizados abusivamente a pesar de que los Gobiernos estaban legitimados para ejercer las acciones penales pertinentes. Es más, los tribunales arbitrales en los casos City Oriente, Quiborax y Lao Holding reconocieron el derecho del Estado de iniciar los juicios contra los responsables, como también el hecho de que no influyen en los procesos arbitrales; no obstante, no es admisible su uso para fines ajenos que perjudiquen al demandante ${ }^{23}$. Es decir, hablamos de una modalidad distinta de prevenir perjuicios de las partes, principio que se ha repetido en un sinfín de juicios (Burlington con Ecuador ${ }^{24}$; Holiday Inns con Marruecos ${ }^{45}$; Amco con Indonesia ${ }^{26}$ ); lo sancionado sería la instrumentalización del proceso penal para perjudicar la posición procesal del demandante.

En consecuencia, la jurisprudencia internacional basada en el Convenio CIADI respaldaría la suspensión de procedimientos penales domésticos en arbitrajes internacionales de inversión en casos puntuales. La principal razón de ello sería evitar los posibles perjuicios o alteraciones en los derechos de las partes en el arbitraje, ya que sería un procedimiento que pudiera influir no en el resultado, sino en la tramitación y buen desarrollo del mismo.

\section{3. ¿VULNERACIÓN a La SOBERANÍA?}

El contexto citado indica que, en procedimientos arbitrales sometidos al Convenio CIADI, el demandante podría solicitar la suspensión de procesos penales domésticos tramitados dentro del Estado demandado si afectan en alguna medida el resultado del arbitraje. ¿Implica la paralización de dichos procedimientos una vulneración al principio de soberanía estatal? ${ }_{2}$ Es posible establecer que dichas medidas cautelares puedan ser entendidas como una injerencia de un tribunal internacional en el funcionamiento ordinario de un poder estatal?

42 Quiborax S.A., Non Metallic Minerals S.A. y Allan Fosk Kaplún con Estado Plurinacional de Bolivia, párrafos $\mathrm{I}_{4}^{2}-\mathrm{I} 43$.

43 Así, en City Oriente con República de Ecuador, párrafo 62; Quiborax S.A., Non Metallic Minerals S.A. y Allan Fosk Kaplún con Estado Plurinacional de Bolivia, párrafo I2r; Lao Holdings N.V. con República Democrática Popular de Laos, párrafo 25; y Tokios Tokelés con Ucrania, párrafo I6. En este último, si bien no lo dice directamente, se puede deducir al permitir investigaciones en contra de los demandantes.

44 Burlington con Ecuador, caso CIADI RB/08/5, orden procesal I, 29 de junio de 2009 , párrafos 6I-68.

45 Holiday Inns S.A. y otros con Kingdom of Morocco, caso CIADI ARB/72/I, orden, 2 de julio de I 972 .

46 Amco Asia con Indonesia, decisión sobre medidas provisionales, 9 de diciembre de I983, ICSID Reports, I993, p. $4^{\mathrm{I} 2 .}$ 


\section{I. Por qué vulneraría la soberanía de un estado}

Antes de responder, debemos tener en consideración dos ideas preliminares que, en principio, apoyarían la posición de que este tipo de suspensiones serían más una intromisión o limitación al poder estatal establecido que una medida preventiva a favor de los intereses de una parte.

En primer lugar, es posible mantener procedimientos paralelos mientras no influya uno en otro. Sobre todo en el ámbito internacional, es un asunto relativamente pacífico que las esferas de litigación internacional y nacional se encuentran separadas en contextos distintos, por lo que debiera en principio influir uno con otro ${ }^{47}$. Ello se puede ver a partir del principio de agotamiento de remedios nacionales, que evita precisamente la duplicidad de procedimientos sobre una misma materia discutida en sede internacional. Luego, solo se restringe a las partes el derecho a reclamar ante tribunales locales si la pretensión implica una intromisión en la litis del tribunal internacional en la que, por cierto, aquella discusión o fue resuelta o las partes renunciaron a dicho derecho ${ }^{48}$. A contrario sensu, todo otro procedimiento es válido. De este modo, no se justificaría la suspensión de los procedimientos penales toda vez que el conflicto, en principio, no guarda vínculo alguno con las inversiones de una empresa. Son materias opuestas que no influyen una de otra.

En segundo lugar, este tipo de medidas conculcaría el derecho a la tutela judicial, definido por Bordalí como el derecho a acceder a los tribunales y obtener una decisión que resuelva el conflicto ${ }^{49}$. La medida sería una limitación al acceso a la protección por parte de los tribunales nacionales, llamados a ser los que dirimen los conflictos entre ciudadanos; función por cierto amparada en el derecho internacional conforme los artículos 8 de la Declaración Universal de Derechos Humanos y 8 del Pacto de San José, que consagran el derecho a dar acceso a un tribunal. Es decir, otorgar una medida cautelar que paraliza un proceso penal podría traducirse en una denegación a la protección efectiva de intereses jurídicos que son dignos de protección al alero del derecho ${ }^{50}$.

47 McLahan, Shore y Weiniger, International Investment, , 100.

48

Perenco Ecuador Ltd. con República of Ecuador y Empresa Estatal Petróleos del Ecuador (Petroecuador), ICSID caso ARB/o8/6, decisión sobre medidas provisionales, 8 de mayo de 2009 , párrafo 6r.

49 Andrés Bordalí Salamanca, "Análisis crítico de la jurisprudencia del Tribunal Constitucional sobre el derecho a la tutela judicial". Revista Chilena de Derecho 38, n. $^{\circ}$ (20II): $3^{2 I}$.

50 Alejandro Romero Seguel, Curso de derecho procesal civil: La acción y la protección de los derechos (Santiago: Jurídica de Chile, 2006), go. 
Además, cabe recordar que el acceso y funcionamiento del aparato punitivo estatal es un "deber" del Estado en relación con sus ciudadanos, muchas veces siendo el llamado a implementar el proceso penal como instrumento de aplicación del poder punitivo. El Estado es el principal interesado en su promoción, toda vez que busca la ejecución del derecho penal conforme la normativa penal vigente, en forma imparcial y proporcional ${ }^{51}$. $\mathrm{El}$ acceso y funcionamiento del proceso penal y la intervención de todos sus protagonistas gozan de un claro interés público que el Estado debe garantizar como parte de sus atribuciones. Ante una premisa de esta envergadura, la paralización de cualquier proceso penal conllevaría la privación de este "deber".

\subsection{Consecuencias de eventual vulneración de soberanía}

En forma sucinta, los párrafos anteriores justificarían una posible vulneración de la soberanía de un Estado. En la práctica, la paralización de procesos penales implicaría limitar las facultades y prerrogativas de un Estado en cómo manejar su sistema de ajusticiamiento criminal $^{52}$. A primera vista, sería una intervención cuestionable, sobre todo si consideramos que el Convenio CIADI es un mecanismo de resolución de conflictos en torno a inversiones extranjeras, no un mecanismo de control sobre los Estados. Regula pleitos en los que el objeto del juicio son derechos patrimoniales.

En sede penal, la suspensión de dichos procesos implica una carga anómala para el funcionamiento de un Estado, pues restringe su principio de autorregulación. Este principio consiste en que los Estados tienen pleno poder para regular sus propios asuntos, por lo que está vedado a otros Estados inmiscuirse en ellos. Ante esa premisa, con menor razón individuos privados pueden intervenir en asuntos como el ejercicio jurisdiccional, toda vez que existe una relación de superioridad del Estado frente al individuo en el derecho internacional ${ }^{53}$. La admisión de suspender dichos procesos implicaría un cambio en las relaciones los Estados, en la que el primero dejaría de gozar ciertos privilegios ante privados en las relaciones internacionales, como las inmunidades internacionales ${ }^{54}$. En consecuencia, permitir este tipo de medidas cautelares implicaría una suerte de "igualdad" entre los litigantes del arbitraje internacional de inversión extranjera; "igualdad" de partes que conllevaría consecuencias perjudiciales para el desempeño de los órganos de un Estado.

51 Andrés Bordalí Salamanca, "La acción penal y la víctima en el proceso penal”. Revista de Derecho de la Pontificia Universidad Católica de Valparaíso 37 (segundo semestre 20II): 523-524.

52 Rodrigo Gil Ljubetic, "ICSID Provisional Measures to Enjoin Parallel Domestic Litigation", World Arbitration \& Mediation Review 3 (2009): 3 .

53 A. F. M. Maniruzzaman, "Protection in International Investment Arbitration: Challenge to State Sovereignty?" En Interim and Emergency Relief in International Arbitration, ed. por Diora Zinyaeva (Nueva York: Juris, 2015), 7 .

54 Maniruzzaman, "Protection in International", 7. 
En suma, la posibilidad de someter al Estado a dichas medidas en principio implicaría alterar los planes de desarrollo libres de un Estado. Bajo una mirada distinta, sería someter un interés público ante uno privado, como lo es el dirigir la actividad jurisdiccional. Además, el forzamiento de dichas medidas llevaría el peligro de someter la voluntad soberana a las pretensiones de un privado ${ }^{55}$. Por ende, este tipo de medidas estarían en entredicho.

\section{UNa JUSTificación Plausible: PREVenir el abuso del PRoceso}

Al sopesar estos argumentos, la supuesta vulneración o intervención de un tribunal arbitral sometido a la normativa del Convenio CIADI en el funcionamiento ordinario del aparato judicial es, a mi juicio, aparente. Si bien es cierto que la suspensión de un proceso penal puede traer efectos indeseados, no debemos olvidar que las medidas precautorias per se generan una molestia, que se traduce en limitar el ejercicio de ciertos derechos. Ahora bien, esa limitación busca un fin plausible, como es proteger los intereses legítimos de una de las partes, mas no intervenir en los asuntos internos de un Estado. El control de la medida es fundamental, por lo que el tribunal debe velar activamente por el mismo al prevenir que una duración desmesurada de la medida perjudique la posición del afectado.

Por otro lado, debemos recordar que las medidas cautelares decretadas se constituyen como resultado de aceptar la competencia del tribunal arbitral. Debido a que las partes resolvieron someter el conflicto ante un tercero imparcial, ello implica una verdadera autolimitación acordada de la soberanía estatal. No olvidemos que el arbitraje es el resultado de un verdadero consentimiento otorgado por el Estado receptor de las inversiones - muchas veces, aunque no necesariamente, contenido en el tratado de inversión internacional bilateral $^{56}$-, el cual consiente en someter el conflicto a un tribunal.

El Estado otorga jurisdicción al tribunal para resolver los conflictos relativos a inversiones en su territorio, para lo cual está legitimado por el mismo ${ }^{57}$. Luego, si los procesos penales afectan los derechos del demandado o el arbitraje mismo, o incluso la integridad del juicio, conforme el artículo 47 del CIADI, el tribunal está habilitado para conocer y resolver las medidas cautelares solicitadas, incluso la suspensión de procesos penales ${ }^{58}$. Es decir, el

55 Maniruzzaman, "Protection in International", , Io.

56 Por ejemplo, ha sido fallado que el consentimiento por un Estado puede ser otorgado mediante la ley. Es el caso de SPP con Egipto, en el que el consentimiento fue dado por ley. Para más información, véase Lim, Ho y Martins, International Investment, 89-92.

57 Lim, Ho y Martins, International Investment, $57^{-5} 5^{8}$.

58 Así de hecho fue declarado en los casos Tokios Tokéles con Ucrania, párrafo II, y en el caso Caratube International Oil Company LLP con República de Kazajistán, caso CIADI ARB/o8/12,

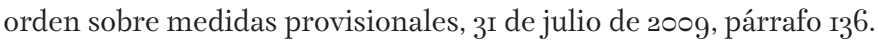


mismo Estado entregó esa posibilidad al acordar las bases del procedimiento y la posibilidad de decretar medidas cautelares. En consecuencia, la suspensión no es precisamente una vulneración a la soberanía, sino otra forma de protección procesal acordada por el mismo Estado en la constitución del tribunal que consintió en limitar. Negar lo anterior sería destruir el sistema de consenso para la existencia del tribunal arbitral y el acuerdo de respetar su jurisdicción.

Volviendo a la suspensión de juicios penales, la medida en sí es excepcional y compleja. Los tribunales arbitrales sometidos al Convenio CIADI exigen un estándar en extremo alto al momento de ordenar la suspensión de un proceso penal. Conforme dicha normativa, el umbral de estándar que debe superar cualquier moción consiste, según la jurisprudencia del CIADI, en que se generará un daño significativo o riesgo ta ${ }^{59}$ que alterará la situación jurídica de las partes si la medida no es acogida ${ }^{60}$. Si bien el estándar es común para toda medida cautelar, el tribunal debe ser especialmente cuidadoso en su otorgamiento, por lo que no bastaría un simple análisis legal, sino también ponderar los efectos colaterales e intensidad de la misma. La suspensión de cualquier proceso - sea doméstico o internacional-implica la postergación de un derecho en disputa, lo que conlleva la definición de un derecho. Ante dicho escenario, además de analizar las circunstancias jurídicas, el tribunal perentoriamente deberá también sopesar las consecuencias de su aplicación: por un lado, la protección de los derechos e intereses legítimos de las partes, mientras que por otro el funcionamiento ordinario del Estado como principal protagonista del proceso penal.

En este caso, si un proceso penal es llevado conforme las prerrogativas y principios que lo inspiran, no es plausible su paralización. Ahora bien, si su génesis obedece para la concreción de fines diversos, como perjudicar la posición jurídica del demandante en el arbitraje internacional, hacer ilusoria la ejecución de los derechos exigidos o conculcar algún derecho procesal de las partes, la suspensión resulta imperiosa.

Así, es posible configurar que la suspensión de un procedimiento penal en un arbitraje regido por el Convenio CIADI podría obedecer a la prevención del daño que de forma natural se condice si su pausa no es decretada. De este modo, solo podría ser suspendido el proceso si este genera un perjuicio intencional del Estado a las pretensiones del demandante o sus derechos durante el procedimiento. Como señaló el tribunal arbitral en el caso Hydro S.r.l. y otros con Albania, lo que busca la suspensión es la pausa, no la eliminación del procedimiento, de modo que terminado el arbitraje este puede seguir su desarrollo natural ${ }^{61}$. Ello no conllevaría una alteración de la soberanía estatal, sino una mera postergación hasta finalizado el pleito.

59 Sarooshi, "Provisional Measures", 377.

60 Caratube International Oil Company LLP con República de Kazajistán, párrafo I37.

61 Hydro S.r.l. y otros con Albania, caso CIADI ARB/I5/28, orden de medidas provisorias, 3 de marzo de 2016 , párrafo 3.20 . 
Todo lo anterior lleva a explicar que los procesos penales son susceptibles de suspensión si son utilizados por el Estado para dañar la posición procesal del demandante u obtener alguna ventaja indebida. Así, es posible fundar los casos de suspensión de procedimientos penales conocidos en procesos sometidos al Convenio CIADI en torno al principio del abuso del proceso. Podemos describir el abuso del proceso como aquella conducta que busca utilizar las reglas procesales para fines completamente diversos a aquellos que intrínsecamente persigue un proceso, como es la solución de una controversia, cuyos resultados son ilícitos o no son posibles de amparar por el sistema jurídico ${ }^{62}$. Es una conducta desleal y arbitraria cuyo fin exclusivo es la afectación de derechos de la contraria. Lo relevante es que exista una afectación a algún derecho relacionado con el proceso - como el derecho a acceder al proceso, debido proceso y derecho a la prueba- de modo que requiera la tutela inmediata del tribunal.

\section{DOS CIRCUNSTANCIAS SOBRE LA SUSPENSIÓN DEL PROCESO PENAL EN PROCESOS SOMETIDOS AL CONVENIO CIADI}

El principio rector de la suspensión del proceso penal como medida cautelar en los arbitrajes internacionales sometidos a la normativa CIADI sería el abuso del proceso. En pocas palabras, el proceso penal perjudica a una de las partes al ser instrumentalizado por el Estado demandado, cuyo fin generalmente será dañar al demandante. Este daño puede ser desincentivar la prosecución del arbitraje por parte del demandante, obstaculizar la obtención o práctica de medios probatorios del inversionista demandante, o generar pruebas a favor del Estado demandado, entre otros.

Ahora bien, la suspensión de las medidas cautelares en el arbitraje internacional de inversiones extranjeras sujeto al Convenio CIADI nos lleva a analizar dos circunstancias inherentes al mismo. La primera, respecto de la prueba de la intención de utilizar de forma abusiva el proceso criminal, toda vez que el ejercicio de una acción penal no implica de inmediato un acto que busque perjudicar la situación procesal del demandante en el arbitraje. La segunda se relaciona con la ejecución de estas medidas cautelares, en concreto, la eficacia de ordenar al Estado demandado el no perseverar en el proceso penal objeto de la suspensión. Ambos factores son relevantes en la práctica arbitral, toda vez que miden la posibilidad tanto de su otorgamiento como la factibilidad de su ejecución.

\section{I. Prueba del abuso del proceso}

Al hablar del abuso procesal en un arbitraje CIADI, surge la duda razonable de cómo probar si el inicio de un procedimiento penal es ejercido en forma abusiva o no por un Estado. Esto es particularmente delicado si consideramos que el derecho no es una dis-

62 Michele Taruffo, "Abuso de proceso", Revista de la Maestría en Derecho Procesal 6, n. ${ }_{2}$ (2016): I6-I7. 
ciplina moralizante, en especial el derecho arbitral o procesal ${ }^{63}$, en los que el inicio de cualquier proceso no solo no implica un abuso per se, sino que es un derecho de acceso a la justicia. Bastaría con cumplir con las normas mínimas para que la conducta mantenga un velo de justificación.

En cuanto a qué debe ser probado, a nuestro juicio son dos los elementos relevantes que requieren ser acreditados, además de la necesidad y urgencia de la medida. En primer lugar, que la conducta genera una afectación desproporcionada en los derechos del demandante. Es decir, que existe un daño palpable de la posición originaria procesal de una parte. Luego, si esa conducta lesiva no existiera, las partes probablemente gozarían de los mismos derechos que al momento de iniciar el juicio. Lo relevante no es que exista un daño per se, sino que este sea completamente anormal y generado a partir de ese proceso.

En segundo lugar, debe probarse la intención de instrumentalización del proceso, con un fin contrario a los fines del proceso ${ }^{64}$. En este ámbito, el abuso no se encuentra en si el ejercicio del derecho genera un daño, sino en si la dirección del daño busca exclusivamente menguar la posición jurídica de las partes, sea que haya iniciado el proceso o incluso en pos de debilitarlo ante un posible arbitraje. Esta intención debe ser concreta y precisa, por lo que es posiblemente el elemento más complejo de probar.

Ahora bien, los casos comentados dan indicios de cómo es posible indagar aquella intención. En efecto, en cuatro de los cinco casos el tribunal tuvo por acreditado que el Estado utilizó el proceso penal para fines aislados a los presupuestados, o al menos su intención difiere al ejercicio de la acción penal. En el caso de City Oriente, el tribunal razonó que el proceso iniciado por los órganos de persecución penal tenía como fin presionar el pago de regalías que, justamente, eran cuestionadas por la demandante del juicio arbitral. El mismo tribunal decretó que, a pesar de ser un procedimiento válido en la forma, su objetivo era diverso: presionar de manera indebida el pago de esos impuestos cuestionado ${ }^{65}$. Es probable que el tribunal tuvo en consideración no solo que el proceso tenía un fin ajeno, sino que además afectaría la posición de cuestionar la situación jurídica del demandante y su estrategia procesal, toda vez que pagar sería un reconocimiento tácito de la legalidad del impuesto. Luego, la demanda de indemnización por trato injusto perdería fuerza.

Misma situación se describe en Quiborax S.A., Non Metallic Minerals S.A. y Allan Fosk Kaplún con Bolivia, en el que el tribunal consideró que los procesos penales llevados contra personeros de la empresa demandante tenían como fin principal no la persecución de hechos punibles, sino mermar la prueba de esta última, afectando su calidad procesal inicial

\footnotetext{
63 Taruffo, "Abuso de proceso", 6-7.

64 Ana Carolina Trigiani, "El Abuso procesal”, Aequitas Virtual II, n. ${ }^{2} 27$ (20I7): 5.

65 City Oriente con República de Ecuador, párrafos 6I-62.
} 
en forma intencional. Es más, señala con claridad que los procesos penales llevados por el Estado boliviano fueron iniciados debido al arbitraje iniciado por la demandante ${ }^{66}$. En el caso Nova, la intención del Estado era precisamente seguir con el proceso de extradición para disminuir una prueba en extremo importante como era la declaración del representante de la demandante. Tanto así, que el tribunal estimó que debido a la alta posibilidad de que el representante podía quedar privado de libertad, era un "riesgo cierto" la pérdida de ese medio probatorio si la medida no era decretada ${ }^{67}$. En Lao Holdings con Laos, el tribunal arbitral descubrió la intención abusiva del procedimiento, toda vez que el mismo Estado confesó en uno de sus escritos de discusión que podría utilizar la información recolectada de los procedimientos penales para mejorar su posición procesal. Luego, el tribunal debió restringir la facultad del Estado en perseguir las responsabilidades penales contra personeros de la demandante, toda vez que la intención de su actuar fue admitido ${ }^{68}$.

Finalmente, en Tokios Tokelés con Ucrania, si bien el proceso penal iniciado por el Estado ucraniano no fue suspendido por diferencias fácticas que impedían deducir una intención de perjudicar a la demandante, el tribunal declaró que estaba facultado para suspender procesos penales si se instrumentalizan para fines ajenos al juicio.

A partir de estos casos, es posible sustraer que todos comparten, en mi opinión, un principio común: debe acreditarse un elemento intencional adicional a la urgencia y necesidad de la medida invocada. Los casos analizados, en sus propias circunstancias, coinciden en que el proceso penal no puede ser un medio en sí mismo para fines con resultados perjudiciales para la contraparte. Ello significa que el elemento volitivo es esencial de acreditar, lo que complejiza en cierto modo la posibilidad de suspender dichos procesos. El demandante deberá acreditar en forma fehaciente no únicamente el daño, sino la intención fraudulenta del proceso penal, cuestión que solo podrá, en muchos casos, ser comprobado mediante indicios. Ejemplo de ello sería el caso de Quiborax S.A., Non Metallic Minerals S.A. y Allan Fosk Kaplún con Bolivia, en que el hecho de que el proceso comenzó con posterioridad al arbitraje CIADI, así como el que los delitos invocados tenían directa relación con inutilizar los medios de prueba de la demandante, son un potente indicio de abuso del proceso.

Ahora bien, todo lo anterior se encuentra en la línea de la preservación de los derechos del demandante y el principio de soberanía de los Estados. Si el proceso se sujeta a sus fines

66 Quiborax S.A., Non Metallic Minerals S.A. y Allan Fosk Kaplún con Estado Plurinacional de Bolivia, párrafo I2r. Expresamente, señala: "Además, aunque el Tribunal tiene absoluto respeto por el derecho soberano de Bolivia de iniciar acciones penales por delitos cometidos dentro de su territorio, las pruebas presentadas sugieren que el proceso penal se inició como consecuencia de una inspección dirigida a los demandantes debido a que habían iniciado este arbitraje”.

67 Nova Group Investments, B.V. con Romania, párrafo 290.

68 Lao Holdings N.V. con República Democrática Popular de Laos, párrafos 27-28. 
intrínsecos, este debe seguir con su curso normal. Sin embargo, si no se ajusta al mismo, se trata de procesos que en sí son conductas contrarias a la buena fe y el uso responsable de los derechos ${ }^{69}$. La conducta del Estado en los casos analizado era abusiva y perjudicial en los derechos de la demandante, estando el tribunal obligado a suspenderlo.

\subsection{Ejecución de la medida cautelar}

El segundo problema planteado es el caso de ejecutar la medida cautelar de suspensión dictada por el tribunal arbitral ante un tribunal local del Estado huésped de las inversiones. Es probable que una medida de esta envergadura sea resistida por el Estado demandado. Ahora bien, zpuede el demandante ejecutar la resolución emitida por el tribunal arbitral contra el mismo Estado demandado?

En principio, debería ejecutarse. Conforme el artículo 47 del CIADI y el artículo 39 de su Reglamento, establecen en su versión en español que el tribunal podrá "recomendar" (en inglés, recommend) las medidas cautelares en cuestión. En un principio, esto significó un debate acerca de la viabilidad de ejecutar las medidas contra el Estado que promueve el proceso penal, toda vez que no sería del todo claro si son vinculantes y, en consecuencia, susceptibles de ejecutar ${ }^{70}$. Actualmente, esta discusión se encuentra más que zanjada desde el caso Emilio Agustín Maffezini con Reino de España, toda vez que se resolvió que la palabra "recomendar", a lo largo del Reglamento y Convención del CIADI, era sinónimo de la palabra "ordenar", junto con el hecho de que si es imperativo el cumplimiento del laudo arbitral, con mayor razón también las resoluciones recaídas en medidas cautelares ${ }^{71}$. Ahora, ello no implica que la medida será inmediatamente ejecutada. Si bien los casos de desacato de Estados son escasos, ellos existen ${ }^{72}$.

Luego, las soluciones para evitar dichos desacatos no siempre son efectivas. Una de ellas es, en caso de que el Estado no obedeciera lo ordenado por el tribunal, el derecho del in-

69 Pía Tavolari Goycoolea, El abuso en el proceso (Santiago: Congreso, 2004), 124.

70 Maniruzzaman, "Protection in International", II-I2.

71 Emilio Agustin Maffezini con Reino de España, caso CIADI ARB/97/7, decisión sobre medida provisoria, 28 de octubre de i999, párrafo 9 .

72 Un ejemplo es el caso Hydro S.r.l. y otros con Albania. Si bien el tribunal arbitral resolvió suspender dos órdenes de detención emitidas por los tribunales de Albania en contra de dos de los demandantes, este último no acató lo ordenado. Es más, Albania siguió con los procesos penales en contra de ambos demandantes, llegando a solicitar su detención y extradición al Reino Unido. Finalmente, esto no se llevó a cabo debido a que los mismos tribunales británicos denegaron la extradición, ya que Albania estaba infringiendo la medida cautelar dictada por el tribunal arbitral. Para mayor información, véase Giovanni Zarra, "The Interference of ICSID Provisional Measures with National Criminal Proceedings", The Italian Yearbook of International Law Online 26, n. ${ }^{\circ}$ I (octubre 2017): $98-99$. 
versionista privado a solicitar la intervención de su país en el pleito en virtud del artículo 27 del CIADI. Esta solución es poco útil, ya que nunca se ha dado el caso, hasta hoy, de que el país de residencia del demandante haga tratativas con el demandado. Otra solución es el derecho de denunciar al Estado incumplidor conforme el artículo 64 del CIADI y someter el conflicto ante el Tribunal Internacional de Justicia. Esta solución también es poco práctica, toda vez que su discusión se centrará en la infracción al Convenio CIADI por parte del Estado demandado, lo que desenfocaría el principal conflicto, que es la falta de ejecución de la medida cautelar. A ello debe sumarse que el procedimiento debe ser iniciado por el Estado del país que suscribió el instrumento internacional que ampara las pretensiones del demandante del pleito sometido al Convenio CIADI, no el inversionista privado. Todo lo anterior hace engorroso este mecanismo. A lo sumo, llegar a esas etapas internacionales únicamente perjudicaría el prestigio del Estado demandado ${ }^{73}$.

Por lo anterior, en general los tribunales arbitrales suelen sancionar a los Estados demandados mediante indemnizaciones de perjuicios, seguidos de la prosecución del proceso penal o en costas, ambas establecidas en la sentencia definitiva. No obstante, la solución también es criticable: el proceso penal abusivo interviene muchas veces en los medios de prueba y pretensiones del demandante, por lo que influirá en cierta medida en el resultado del juicio. Por otro lado, la indemnización de perjuicios busca sanear un daño muchas veces difícil de sanear - como ocurre con frecuencia con los efectos del proceso penal-. Además, la condena en costas no es sanción para este tipo de conductas ${ }^{74}$.

Ello deja al descubierto uno de los principales desafíos de las medidas cautelares: la efectividad de ejecutar la medida contra el Estado. Si bien este trabajo no busca dar una solución al respecto, esta es una tarea que debe ser investigada y generar nuevas propuestas. De lo contrario, se seguirá lo que ocurrió en el caso Quiborax: a pesar de la orden del tribunal, Bolivia desestimó la medida cautelar de suspensión del proceso penal en contra de personeros de dicha empresa y siguió con su curso ordinario, lo que generó consecuentes daños en la prueba de la demandante. Misma situación se vivió en el caso Nova: a pesar de la prohibición decretada por el tribunal, Rumania prosiguió con el proceso de extradición de Adamescu, cuya residencia se encontraba en Londres. Incluso más: dicha extradición fue luego acogida por los tribunales ingleses, en una muy cuestionable sentencia ${ }^{75}$. Ambos

73 Zarra, “The Interference”, 99.

74 Zarra, "The Interference", IoI.

75 Una excelente crítica a la sentencia dictada por la Corte de Westminster puede ser encontrada en Emily Gonini, "How Effective are ICSID Provisional Measures at Suspending Criminal Proceedings before Domestic Courts: The English Example?", Kluwer Arbitration Blog, $3 \circ$ de septiembre de 2017 , http://arbitrationblog.kluwerarbitration.com/20I7/og/30/effective-icsid-provisional-measures-suspending-criminal-proceedings-domestic-courts-english-example/. 
casos se traducen en una especie de autotutela por una de las partes, quien deja en sus manos decidir si considerar el proceso como abusivo a no. Ello no solo afecta el resultado del litigio, sino que deja en entredicho la capacidad e idoneidad del tribunal de ejecutar sus resoluciones y el sistema de arbitraje internacional de inversión extranjera del CIADI como método de solución de controversias efectivo.

\section{CONCLUSIón}

La suspensión de procesos penales por orden de un tribunal arbitral ajeno al conminado a llevar el procedimiento suspendido es de particular complejidad. Ello implica no solo la postergación de resolver un procedimiento, sino que limita un derecho fundamental, como es el acceso a tribunales, y pone en tela de juicio los límites de la soberanía estatal. Su suspensión significa limitar una fracción del poder soberano de un Estado en la persecución de hechos constitutivos de delitos y el cumplimiento de uno de sus deberes legales y, muchas veces, constitucionales.

Sin embargo, excepciones a la regla general existen, y este caso es uno de aquellos. Los casos analizados demuestran que, bajo ciertas circunstancias, los tribunales arbitrales internacionales sometidos al Convenio CIADI tienen jurisdicción para suspender procedimientos penales nacionales. Esta medida debe cumplir un estándar exigente, del cual es el principio rector de suspensión el uso indebido del proceso para fines diversos. Más que una vulneración a la soberanía del Estado, lo anterior parece ser una facultad encaminada a evitar perjuicios conforme a las prerrogativas entregadas por el mismo Estado en las bases arbitrales, en las que entrega al tribunal las facultades necesarias para dirimir el conflicto conforme un debido proceso. Ello no es más que el reflejo del principio de

sometimiento a resolver los conflictos en forma pacífica de conformidad al artículo 25 del 


\section{BIBLIOGRAFÍA}

- Bjorklund, Andrea. "State Immunity and the Enforcement of Investor-State Arbitral Awards". En International Investment Law for the 21st Century: Essays in Honour of Christoph Schreuer, por Christina Binder, Ursula Kriebaum, August Reinisch y Stephan Wittich. Oxford: Oxford University Press, 2009.

- Bordalí Salamanca, Andrés. "Análisis crítico de la jurisprudencia del Tribunal Constitucional sobre el derecho a la tutela judicial". Revista Chilena de Derecho 38, n. ${ }^{\circ} 2$ (2011): 311-337. doi: 10.4067/S071834372011000200006 .

- - "La acción penal y la víctima en el proceso penal". Revista de Derecho de la Pontificia Universidad Católica de Valparaíso 37 (segundo semestre 2011): 513-545. doi: 10.4067/S0718-68512011000200013.

- Commission, Jeffery y Rahim Moloo. Procedural Issues in International Investment Arbitration. Oxford: Oxford University Press, 2018.

- Cortez Matcovich, Gonzalo. "Contribución al estudio de las medidas cautelares previas a la demanda en el proceso civil chileno". Revista de Derecho 30, n. ${ }^{\circ} 1$ (junio 2017): 235-261. doi: 10.4067/S071809502017000100010.

- Crawford, James. Brownlie's Principles of Public International Law. 8. a edición. Oxford: Oxford University Press, 2012.

- Douglas, Zachary. "The Hybrid Foundations of Investment Treaty Arbitration". The British Yearbook of International Law 74, n. ${ }^{\circ} 1$ (noviembre 2004): 151-289. doi: 10.1093/bybil/74.1.151.

- Gazzini, Tarcisio y Robert Kolb. "Provisional Measures in ICSID Arbitration from 'Wonderland's Jurisprudence' to Informal Modification of Treaties". The Law \& Practice of International Courts and Tribunals 16, n. ${ }^{\circ} 1$ (junio 2017): 159-184. doi: 10.1163/15718034-12341346.

- Gil Ljubetic, Rodrigo. "ICSID Provisional Measures to Enjoin Parallel Domestic Litigation”. World Arbitration \& Mediation Review 3 (2009).

- Jellinek, Georg. Teoría general del Estado. Buenos Aires: Albatros, 1978.

- Lim, Chin Leng, Jean Ho y Martin Paparinskis. International Investment Law and Arbitration. Cambridge: Cambridge University Press, 2018. 
- Maniruzzaman, A. F. M. "Protection in International Investment Arbitration: Challenge to State Sovereignty?" En Interim and Emergency Relief in International Arbitration, editado por Diora Zinyaeva. Nueva York: Juris, 2015.

- McLachlan, Campbell, Laurence Shore y Matthew Weiniger. International Investment Arbitration. 2. ${ }^{\text {a }}$ edición. Oxford: Oxford University Press, 2017.

- Nieva, Jordi. Derecho procesal I: Introducción. Madrid: Marcial Pons (2014).

- Ried Undurraga, Ignacio. "Tres cuestiones sobre la litispendencia en el proceso civil chileno". Revista de Derecho de la Pontificia Universidad Católica de Valparaíso 45 (segundo semestre 2015): 205-241. doi: 10.4067/S0718-68512015000200008.

- Ríos Álvarez, Lautaro. "La soberanía, el poder constituyente, y una nueva Constitución para Chile". Estudios Constitucionales 15, n. ${ }^{\circ} 2$ (2017): 167-202. doi: 10.4067/S0718-52002017000200167.

- Romero Seguel, Alejandro. Curso de derecho procesal civil: La acción y la protección de los derechos. Santiago: Jurídica de Chile, 2006.

- -. Curso de derecho procesal civil. Tomo 3. Santiago: Thomson Reuters, 2015.

- Sarooshi, Dan. "Provisional Measures and Investment Treaty Arbitration". Arbitration International 29, n. ${ }^{\circ} 3$ (septiembre 2013): 361-380. doi: 10.1093/arbitration/29.3.361.

- Shaw, Malcolm. International Law. 8. ${ }^{\mathrm{a}}$ edición. Cambridge: Cambridge University Press, 2017.

- Taruffo, Michele. "Abuso de proceso". Revista de la Maestría en Derecho Procesal 6, n. 2 (2016): 6-29. Recuperado de http://revistas.pucp. edu.pe/index.php/derechoprocesal/article/view/16417.

- Tavolari Goycoolea, Pía. El abuso en el proceso. Santiago: Congreso, 2004.

- Trigiani, Ana Carolina. "El abuso procesal”. Aequitas Virtul 11, n. ${ }^{\circ} 17$ (2016). Recuperado de https://p3.usal.edu.ar/index.php/aequitasvirtual/article/view/4321.

- Zarra, Giovanni. "The Interference of ICSID Provisional Measures with National Criminal Proceedings". The Italian Yearbook of International Law Online 26, n. ${ }^{\circ} 1$ (octubre 2017): 537-547. doi: 10.1163/22116133-90000159. 


\section{JURISPRUDENCIA CITADA}

\section{INTERNACIONAL}

- Abaclat y otros con República Argentina. Caso CIADI ARB/07/5, orden procesal 11, 27 de junio de 2012.

- Amco Asia con Indonesia. Decisión sobre medidas provisionales, 9 de diciembre de 1983, ICSID Reports, 1993.

- Biwater Gauff (Tanzania) Ltd con Tanzania. Caso CIADI ARB/05/22, orden procesal 3, 29 de septiembre de 2006.

- Burlington con Ecuador. Caso CIADI ARB/08/5, orden procesal 1, 29 de junio de 2009.

- Caratube International Oil Company LLP con República de Kazajistán. Caso CIADI ARB/08/12, orden sobre medidas provisionales, 31 de julio de 2009.

- Ceskoslovenska Obchdni Bank A.S. con República de Eslovaquia. Caso CIADI ARB/97/4, decisión del tribunal sobre objeción de jurisdicción, 24 de mayo de 1999.

- City Oriente con República de Ecuador. Caso CIADI ARB/06/21, resolución a solicitud de demandante por medida cautelar, 19 de noviembre de 2007.

- Emilio Agustin Maffezini con Reino de España. Caso CIADI ARB/97/7, decisión sobre medida provisoria, 28 de octubre de 1999.

- Hydro S.r.l. y otros con Albania. Caso CIADI ARB/15/28, orden de medidas provisorias, 3 de marzo de 2016.

- Holiday Inns S.A. y otros con Reino de Marruecos. Caso CIADI ARB/72/1, orden, 2 de julio de 1972.

- Lao Holdings N.V. con República Democrática Popular de Laos. Caso CIADI ARB (AF)/12/6, 17 de septiembre 2013. Decisión sobre medida cautelar, 17 de septiembre de 2013.

- Lao Holdings N.V. con República Democrática Popular de Laos. Caso CIADI ARB (AF)/12/6, decisión para modificar medida cautelar, 30 de mayo de 2014.

- Nova Group Investments, B.V. con Romania. Caso CIADI ARB/16/19, decisión sobre medidas provisionales núm. 7, 29 de marzo de 2017. 
- Perenco Ecuador Ltd. con República de Ecuador y Empresa Estatal Petróleos del Ecuador (Petroecuador). Caso CIADI ARB/08/6, decisión sobre medidas provisionales, 8 de mayo de 2009.

- Plama Consortium Limited con República de Bulgaria. Caso CIADI ARB/03/24, orden, 6 de septiembre de 2005.

- Quiborax S.A., Non Metallic Minerals S.A. y Allan Fosk Kaplún con Estado Plurinacional de Bolivia. Caso CIADI ARB/06/2, decisión sobre medidas provisionales, 26 de febrero de 2010.

- Sergei Paushok, CJSC Golden East Co. y CJSC Vostoknftegaz Co. con Gobierno de Mongolia. UNCITRAL, orden sobre medidas cautelares, 2 de septiembre de 2008

- Tokio Tokelés con Ucrania. Caso CIADI ARB/02/18, orden 1, resolución a solicitud de demandante por medida cautelar, 1 de julio de 2003. 